\title{
Audit on intravenous fluid in children at a teaching hospital in North East England
}

\author{
Samath Samitha Somarathna ${ }^{1}$ \\ Sri Lanka Journal of Child Health, 2012; 41(3): 129-131
}

\begin{abstract}
Objectives: To compare the current practice of intravenous fluid regime usage in children from one month to 16 years at University Hospital of North Tees and University Hospital of Hartlepool against local guideline developed using National Patient Safety Agency (NPSA) guideline. A subsidiary objective was to identify desirable changes that need to be done to the existing system.
\end{abstract}

Method: E-discharge letters were perused from $1^{\text {st }}$ January to $31^{\text {st }}$ December 2009. Children who received intravenous fluid were taken and detailed discharge letters were screened. A pretested proforma was used to audit the discharge notes.

Results: During the one year period 62 patients were included. Fifty two percent were males. Twenty eight children were less than one year old, 24 between $1-5$ years, 6 between $6-10$ years and 4 more than 10 years old. Complete clinical data to assess dehydration was not recorded according to audit standard in any of the patients. Clinical diagnoses were gastroenteritis in 14, viral illness in 10 , bronchiolitis in 8 and pyloric stenosis in 6 . Mild dehydration was noted in 52, moderate dehydration in 4 and severe dehydration in 2.

Compliance on the guideline was audited during different stages of management. Forty percent guideline compliance was achieved during immediate resuscitation, $71 \%$ during maintenance fluid, $50 \%$ during fluid deficit correction, $0 \%$ during replacement of on-going losses, 78\% during monitoring of electrolytes and $6 \%$ during monitoring of fluid balance.

Conclusion: This audit showed that audit standards were not achieved at different levels of management.

(Key words: clinical audit, intravenous fluid management)

\footnotetext{
${ }^{1}$ Acting Paediatrician, Lady Ridgeway Hospital for
} Children, Colombo

(Received on 5 October 2011: Accepted after revision on 28 October 2011)

\section{Introduction}

In North East of England, University Hospital of North Tees \& Hartlepool is a major teaching hospital having one hospital at each site. It has a paediatric unit at each hospital.

Local guideline ${ }^{1}$ was developed using National Patient Safety Agency (NPSA) guideline ${ }^{2}$ which is a body of the Department of Health, UK. The guideline consists of five steps:

1. Immediate fluid resuscitation

2. Calculating intravenous fluid daily 'maintenance'

3. Correction of fluid deficits

4. Ongoing losses

5. Monitoring and alteration of fluids

Immediate fluid resuscitation requires $20 \mathrm{ml} / \mathrm{kg}$ of $0.9 \%$ sodium chloride intravenously except in trauma when it is $10 \mathrm{ml} / \mathrm{kg}$. Maintenance requirements were assessed using the Holliday and Segar formula ${ }^{3}$ and fluids were either $0.45 \%$ sodium chloride with $5 \%$ dextrose or $0.9 \%$ sodium chloride with 5\% dextrose. Fluid deficit was assessed using either premorbid weight versus admission weight or by estimation of percentage dehydration using clinical signs. Fluid was $0.9 \%$ sodium chloride with $5 \%$ dextrose and replaced over 24-48 hours. Ongoing losses were corrected over the next 4 hours with the fluid being similar to the lost fluid. Potassium was added for vomiting and diarrhoea with maximum concentration being $40 \mathrm{mmol} /$ litre and given via a separate intravenous line. Minimum monitoring standards included checking serum urea, creatinine and electrolytes before intravenous fluids were started and checking every 24 hours if it was continuing. However, if abnormal results of electrolytes were suspected or seen it was deemed essential to repeat at 4-6 hours. Regular measurement of blood glucose, daily weighing and daily measurement of urine output were part of the monitoring. However, if a sicker child is seen more frequent assessments have to be done. 


\section{Objectives}

1. To compare current practice of intravenous fluid regime in children from 1 month to 16 years, at University Hospital of North Tees and Hartlepool against local guidelines ${ }^{1}$ made using NPSA guidelines ${ }^{2}$.

2. To identify desirable changes that needed to be done to the existing system.

\section{Method}

E discharge letters were perused from $1^{\text {st }}$ January 2009 to $31^{\text {st }}$ December 2009. During the audit period there were 7,416 total admissions. Intravenous fluids were given to 106 patients and only 62 patients were recruited to the audit. Forty four patients were excluded due to presence of exclusion criteria viz. diabetic, renal, endocrine, cardiac, burns, neonates and meningococcal patients.

A pre tested proforma, which was approved by the clinical governance unit of the hospital, was used to conduct the audit. The proforma has sections on demography, clinical data, immediate resuscitation, maintenance fluid, correction of fluid deficit, ongoing losses and monitoring. Each of the sections was assessed according to the audit criteria.

\section{Results}

Demography: Thirty two (52\%) were males and ages were divided as follows; <1year 28 (45\%), 1-5 years $24(38 \%), 6-10$ years $6(9 \%)$ and $>10$ years 4 $(6 \%)$.

Clinical data: Tachycardia was noted in $44(70 \%)$, not seen in $16(25 \%)$ and not recorded in $2(3 \%)$. Reduced pulse volume was noted in $12(19 \%)$, not seen in $16(26 \%)$ and not recorded in $34(55 \%)$. Prolonged capillary refilling time was noted in 24 $(38 \%)$, not seen in $18(29 \%)$ and not recorded in 20 $(32 \%)$. Blood pressure was normal in $18(29 \%)$ and not recorded in 44 (71\%). Tachypnoea was noted in $22(35 \%)$, not seen in $28(45 \%)$ and not recorded in $12(19 \%)$.

Clinical diagnosis: Fourteen (23\%) had gastroenteritis, $10(17 \%)$ had viral illness, $8(14 \%)$ had bronchiolitis, $6(10 \%)$ had pyloric stenosis, 4 (6\%) each had swine flu, asthma and appendicitis and $2(3 \%)$ each had febrile seizures, tonsillitis, pneumonia, otitis media, sepsis and hand-footmouth disease. Mild dehydration was noted in 52 $(84 \%)$, moderate and no dehydration noted in 4 $(6 \%)$ each with severe dehydration in $2(3 \%)$. Methods of rehydration were only intravenous in
$16(26 \%)$ and both intravenous and oral in 46 $(74 \%)$.

Immediate resuscitation was done only in $10(16 \%)$ patients and the type of intravenous fluid used was $0.9 \%$ sodium chloride. Volume of bolus was $20 \mathrm{ml} / \mathrm{kg}$ in $4(40 \%)$ patients which is guideline compliant. However, $10 \mathrm{ml} / \mathrm{kg}$ boluses were used in $4(40 \%)$ and $6 \mathrm{ml} / \mathrm{kg}$ in $2(20 \%)$ patients. Therefore, $40 \%$ guideline compliance was achieved during immediate resuscitation.

Maintenance fluids were given for all 62 (100\%) patients and the type of fluid was $0.45 \%$ sodium chloride with 5\% dextrose. However, addition of potassium chloride was done in only $12(19 \%)$ patients. Therefore, $81 \%$ guideline compliance was achieved during maintenance fluid.

Fluid deficit was corrected only in $4(6 \%)$ patients. Although premorbid weight was available in 2 patients, the dehydration method was used to correct fluid deficit in all 4 patients. However, fluid bolus was subtracted only in 2 patients. Fluids were $0.9 \%$ sodium chloride in $2(50 \%)$ and $0.45 \%$ sodium chloride in the rest. Therefore, $50 \%$ guideline compliance was achieved during fluid deficit correction.

Ongoing losses were present in 6 patients but correction was not done in any of them. In that context, $0 \%$ guideline compliance was noted.

Monitoring included doing serum electrolytes, blood sugar, weighing, measuring urine output and fluid balance. Serum electrolytes were done in 56 (90\%) and were abnormal in 18 (32\%). However, abnormal electrolytes were repeated only in 14 (78\%). Therefore, $78 \%$ guideline compliance was noted during monitoring electrolytes. Fluid balance daily was done only in $4(6 \%)$, not done in $4(6 \%)$ and not recorded in $54(88 \%)$. Therefore, $6 \%$ guideline compliance was achieved during monitoring of fluid balance.

\section{Discussion}

This audit covered most aspects of the guideline. Usage of intravenous fluid is minimal in paediatric patients $(1 \%)$ in this audit. This coincides with the adherence to a guideline locally made according to national standards.

Proper records are necessary in providing optimal patient care and minimising problems of litigation. Using a proforma for clerking at admission helps remind a busy medical officer of all the facts in clinical data that need to be recorded. 
During immediate resuscitation, $20 \mathrm{ml} / \mathrm{kg} \quad 0.9 \%$ sodium chloride is to be given. However, only $40 \%$ of doctors were compliant with this. Further training and revision is mandatory in this respect.

Frequent monitoring is essential clinically and biochemically when a child receives intravenous fluid. This part is lacking according to the audit.

\section{Conclusions}

During the one year audit period guideline compliance was poor at replacing ongoing losses and monitoring the child while on intravenous fluid. Record keeping was also found to be poor and needs addressing.

\section{Recommendations}

- $\quad$ After the audit medical staff was introduced to a weekly case scenario headed by a consultant paediatrician. This included calculation and usage of intravenous fluids to minimise errors. Application of similar case scenario on a regular basis will add to better knowledge, attitude and practices among medical staff.

- Attending Advanced Paediatric Life Support (APLS) or European Paediatric Life Support (EPLS) was made essential to all medical staff following the audit.
- Proper record keeping is essential for both patient care and avoiding litigation. During induction of new doctors, record keeping section was introduced. New proforma for admission notes were introduced including most clinical data to facilitate busy junior doctor.

\section{Acknowledgements}

I thank Dr P. Venugopalan (Paediatric Audit Lead), Dr I. McClean (Paediatric Gastroenterologist), staff of the clinical audit training and all the staff at University Hospital of Northtees \& Hartlepool for their invaluable help.

\section{References}

1. University hospital of Northtees and Hartlepool; Guidelines for administration of intravenous fluid to children aged 1 month to 16 years. $(2007-2010)$

2. National Patient Safety Agency (NPSA), United Kingdom. Alert No. 22, $28^{\text {th }}$ March 2007

3. Holliday MA, Segar WE. The maintenance need for water in parenteral fluid therapy. Pediatrics 1957; 19: 823-32. 